\title{
CRISPR/Cas9-targeted enrichment and long-read sequencing of the Fuchs endothelial corneal dystrophy-associated TCF4 triplet repeat
}

\author{
Nathaniel J. Hafford-Tear, MSc ${ }^{1}$, Yu-Chih Tsai, PhD ${ }^{2}$, Amanda N. Sadan, MSc ${ }^{1}$, \\ Beatriz Sanchez-Pintado, MSc ${ }^{1}$, Christina Zarouchlioti, MRes ${ }^{1}$, Geoffrey J. Maher, $\mathrm{PhD}^{3}$, \\ Petra Liskova, MD PhD ${ }^{1,4}$, Stephen J. Tuft, MD, FRCOphth ${ }^{1,5}$, Alison J. Hardcastle, PhD ${ }^{1}$, \\ Tyson A. Clark, $\mathrm{PhD}^{2}$ and Alice E. Davidson, $\mathrm{PhD} \mathbb{B D}^{1}$
}

Purpose: To demonstrate the utility of an amplification-free longread sequencing method to characterize the Fuchs endothelial corneal dystrophy (FECD)-associated intronic TCF4 triplet repeat (CTG18.1).

Methods: We applied an amplification-free method, utilizing the CRISPR/Cas9 system, in combination with PacBio single-molecule real-time (SMRT) long-read sequencing, to study CTG18.1. FECD patient samples displaying a diverse range of CTG18.1 allele lengths and zygosity status $(n=11)$ were analyzed. A robust data analysis pipeline was developed to effectively filter, align, and interrogate CTG18.1-specific reads. All results were compared with conventional polymerase chain reaction (PCR)-based fragment analysis.

Results: CRISPR-guided SMRT sequencing of CTG18.1 provided accurate genotyping information for all samples and phasing was possible for 18/22 alleles sequenced. Repeat length instability was observed for all expanded ( $\geq 50$ repeats) phased CTG18.1 alleles analyzed. Furthermore, higher levels of repeat instability were associated with increased CTG18.1 allele length (mode length $\geq 91$ repeats) indicating that expanded alleles behave dynamically.

Conclusion: CRISPR-guided SMRT sequencing of CTG18.1 has revealed novel insights into CTG18.1 length instability. Furthermore, this study provides a framework to improve the molecular diagnostic accuracy for CTG18.1-mediated FECD, which we anticipate will become increasingly important as gene-directed therapies are developed for this common age-related and sight threatening disease.

Genetics in Medicine (2019) 21:2092-2102; https://doi.org/10.1038/s41436019-0453-x

Keywords: Fuchs endothelial corneal dystrophy; amplificationfree sequencing; triplet repeat-mediated disease; somatic mosaicism; no-amp targeted sequencing

\section{INTRODUCTION}

Currently there are more than 40 human diseases that are caused by the expansions of simple nucleotide repeat sequences (microsatellites), with diagnosis and prognosis often dependent on accurate sizing of mutant alleles. ${ }^{1}$ Despite the significant advances in sequencing technologies over the past decade, microsatellites are still typically investigated in a diagnostic setting using polymerase chain reaction (PCR)based amplification methods and fragment sizing by capillary electrophoresis. When microsatellites expand they become intractable to standard short-read next-generation sequencing (NGS) technologies due to their innate repetitive nature, size, and typically high GC content. Furthermore, when there are large differences in size between wild-type and mutant expanded alleles, as is often the case for autosomal dominant disorders, skewed allelic amplification efficiencies hinder amplification-based protocols and their analyses. ${ }^{2}$

Fuchs endothelial corneal dystrophy (FECD; OMIM 613267) affects up to $4.5 \%$ of individuals over 50 years of age. $^{3}$ Approximately $75 \%$ of cases harbor a noncoding CTG microsatellite expansion termed CTG18.1, making FECD the most prevalent triplet repeat-mediated disease in humans. ${ }^{4}$ FECD is an age-related, degenerative condition that primarily affects the posterior corneal layers and it is the most frequent indication for corneal transplantation in the developed world. ${ }^{5}$ It is clinically characterized by the accelerated loss of endothelial cells and progressive thickening of Descemet membrane with focal excrescences termed guttae. $^{6}$ In

${ }^{1}$ UCL Institute of Ophthalmology, London, UK; ${ }^{2}$ Pacific Biosciences, Menlo Park, CA, USA; ${ }^{3}$ Clinical Genetics Group, MRC Weatherall Institute of Molecular Medicine, University of Oxford, John Radcliffe Hospital, Oxford, UK; ${ }^{4}$ Department of Ophthalmology, First Faculty of Medicine, Charles University and General University Hospital in Prague, Prague, Czech Republic; ${ }^{5}$ Moorfields Eye Hospital, London, UK. Correspondence: Alice E. Davidson (alice.davidson@ucl.ac.uk)

These authors contributed equally: Nathaniel J. Hafford-Tear, Yu-Chih Tsai 
advanced disease, loss of endothelial cell function leads to corneal edema, progressive corneal opacity, and reduced vision. ${ }^{7}$ Expansion of CTG18.1 situated on Chr18q21.1 within an intron of TCF4 was first shown to be significantly associated with FECD in 2012. ${ }^{8}$ Using a combination of short tandem repeat (STR) assays and Southern blotting the authors demonstrated that $79 \%$ of the patient cohort had at least one expanded copy of the triplet CTG motif (defined as $\geq 50$ repeats) compared with $3 \%$ of control individuals. ${ }^{8}$ This striking association has subsequently been replicated in ethnically distinct populations using comparable methodologies. ${ }^{4,8-10}$ Typically, FECD patients harbor heterozygous expansions of the repeat in the range of 50-200 repeat units; however for a few patients, much larger expansions estimated to be up to several thousand repeat units have been identified by Southern blotting. ${ }^{8,9,11}$

In this study we demonstrate a custom application of an amplification-free long-read sequencing method (BioRxiv: https://doi.org/10.1101/203919) to specifically study the TCF4 repeat element at a nucleotide level. The method, termed noamp targeted sequencing, utilizes the CRISPR-Cas9 system to target, enrich, and isolate desired DNA fragments in a non-amplification dependent fashion. ${ }^{12-14}$ In combination with long-read single-molecule real-time (SMRT) sequencing $^{15,16}$ this approach enabled us to analyze the diseaseassociated tandem repeat at a nucleotide level within a FECD patient cohort.

\section{MATERIALS AND METHODS}

\section{Selection of FECD patient genomic DNA samples and STR} genotyping assay

This study followed the tenets of the Declaration of Helsinki and was approved by the Moorfields Eye Hospital (MEH) ethics committee (09/H0724/25). A diagnosis of FECD was based on the presence of characteristic confluent corneal guttae on slit-lamp biomicroscopy, or a previous history of a corneal transplant for FECD. Written informed consent was obtained from all participants. Genomic DNA samples were initially genotyped for CTG18.1 using a short tandem repeat (STR) assay as described previously. ${ }^{4,8}$ Throughout this study expanded CTG18.1 alleles are defined as comprising $\geq 50$ CTG repeats.

\section{Design of guide RNAs for Cas9 digestion}

DNA sequences surrounding the CTG18.1 locus and the fragile $\mathrm{X}$ syndrome-associated CGG triplet repeat located within FMR1 were used to design Cas9 guide RNAs (gRNAs). gRNAs were formed by duplexing CRISPR RNA (crRNA) with trans-activating crRNA (tracrRNA). Candidate target sequences were generated using an online CRISPR RNA configurator available on the Dharmacon website, and target specificity was checked against the human genome reference sequence (CRISPR Design Tool: https://dharmacon. horizondiscovery.com/gene-editing/crispr-cas9/crispr-designtool/). Final crRNA sequences used for the CRISPR/Cas9 experiment were manually selected to generate a target capture region of approximately $1 \mathrm{~kb}$ from the Cas 9 digestion site to the nearest EcoRI or BamHI digestion site, according to the hg19 reference. crRNA sequence specificity was verified by BLAST search against the human genome and the 3 ' end of the crRNA was designed to be oriented toward the region of interest. crRNAs used are shown below:

TCF4_crRNA sequence: 5'-CAAGAGGCUAUUUACAGC UA-3'

FMR1_crRNA sequence: 5'-AGAGGCCGAACUGGGAUA AC-3'

\section{Amplification-free Cas9-targeted enrichment of the TCF4 and FMR1 loci}

Approximately 5-20 $\mu \mathrm{g}$ of native genomic DNA was fragmented with high-fidelity restriction enzymes KpnI and EcoRV in the presence of calf intestinal alkaline phosphatase (CIP) (New England Biolabs) to first reduce genome complexity. Sample DNA was subsequently fragmented with EcoRI and BamHI (New England Biolabs) and ligated to restriction site-specific hairpin adapters carrying overhangs for EcoRI and BamHI cut sites (5'-GATCATCTCTCTCT TTTCCTCCTCCTCCGTTGTTGTTGTTGAGAGAGAT-3' and 5'-AATTATCTCTCTCTTTTCCTCCTCCTCCGTTGTT GTTGTTGAGAGAGAT-3') to form SMRTbell template libraries using E. coli DNA ligase (New England Biolabs). DNA fragments previously cut with the restriction enzymes KpnI and EcoRV were not compatible with SMRTbell adapters and were subsequently digested by exonuclease.

Next, $1 \mu \mathrm{g}$ of SMRTbell template was digested using $32 \mathrm{nM}$ Cas9 nuclease (New England Biolabs) and $48 \mathrm{nM}$ target specific gRNAs (Integrated DNA Technologies) in $50 \mu \mathrm{l}$ digestion reaction for 1 hour. crRNAs duplexed to tracrRNA at a 1:1 ratio was used for the Cas9 digestion step. Both TCF4 and FMR1 (positive control) gRNAs were multiplexed in the same digestion reaction. PolyA hairpin capture adapters (5'ATCTCTCTCTTAAAAAAAAAAAAAAAAAAAAAAATT GAGAGAGAT-3') were ligated to the DNA molecules targeted by the Cas9 digestion to form a library of asymmetrical SMRTbell template molecules.

DNA molecules, with ligated capture adapters, were enriched using a MagBead system (PacBio). Binding of capture adapters to a MagBead complex was carried out by incubating components in MagBead binding buffer for 2 hours at $4{ }^{\circ} \mathrm{C}$. MagBeads with bound target fragments were then eluted using elution buffer (PacBio) for 10 minutes at $65{ }^{\circ} \mathrm{C}$.

\section{Targeted SMRT sequencing}

Target fragments were prepared for SMRT sequencing by annealing a standard PacBio primer lacking PolyA sequence and incubating at room temperature for 1 hour. Then, $0.6 \times$ AMpure Beads were used to remove unbound primer. DNA molecule/primer complexes were subsequently bound with P6 polymerase in the presence of free hairpin adapters to remove 
excess polymerase. Sequencing was completed using the PacBio RSII instrument with one-cell-per-well MagBead sequencing protocol, $\mathrm{P} 6 / \mathrm{C} 4$ chemistry, and 240-minute run time. Circular consensus sequencing (CCS) reads were generated using PacBio SMRTPortal (version 2.3) software with a $90 \%$ predicted accuracy setting.

\section{Genome-wide coverage plots}

Genome-wide coverage plots were generated for each sample from total CCS reads using PacBio SMRTPortal (version 2.3), in accordance with methods outlined previously (https://doi. org/10.1101/203919).

\section{Alignment, filtering, and base calling of locus-specific CCS reads}

On-target CCS reads were identified by mapping the flanking sequences on either side of the repeat region. Mapped reads were then further filtered to only retain reads with $\geq 90 \%$ similarity to the flanking sequences, irrespective of the CTG18.1 repeat length. On-target CCS read sequences for each sample were then visualized to identify the biallelic CTC repeat lengths, and to determine the maximum CTG18.1 repeat length present per sample. Consensus sequence mapping was also used to identify the genotype of a polymorphic intronic TCF4 SNP situated downstream of CTG18.1 (rs599550) for each allele of each sample.

On-target CCS reads were subsequently mapped to a pool of reference sequences that included allele-specific flanking sequences (CTC repeat lengths, rs599550 genotype) and CTG repeat sizes up to the maximum length previously determined (per sample). Phasing was possible when individuals were either heterozygous for the single-nucleotide polymorphism (SNP) (rs599550) or harbored informative heterozygous CTC repeat lengths. The best matching reference sequence $(\geq 99 \%$ match) for each CCS read was used to infer CTG18.1 repeat length. Frequency histogram plots were generated, using phasing information when possible, to show repeat length reads per sample. For samples that could not be phased, the modal repeat length per local maxima of mapped reads was identified. Mapping was completed using Blasr (https:// github.com/PacificBiosciences/blasr).

FMR1 repeat lengths were determined according the protocol previously described by Hoijer et al. ${ }^{13}$ using the output from a customized R script available from Github (https://github.com/NationalGenomicsInfrastructure/HTTrepeat-analysis).

\section{RESULTS}

\section{Sample selection and amplification-free Cas9-targeted enrichment}

To ascertain whether CRISPR-guided SMRT sequencing could effectively resolve expanded copies of the CTG18.1 locus, whole blood-derived genomic DNA samples from FECD patients with a diverse range of CTG18.1 allele lengths and zygosity status, previously defined by STR genotyping assay, were selected for analysis. ${ }^{4,8}$ In total, 11 DNA samples from the following categories were analyzed: 2 samples with biallelic nonexpanded ( $<50$ repeats) alleles (category A), 5 samples with presumed monoallelic expansions $(\geq 50$ repeats) (category $\mathrm{B}$ ), and 4 samples with presumed biallelic expansions (category C; Table $\mathbf{1}$ ).

An overview of the amplification-free template preparation method employed is presented in Fig. 1a. In brief, the patientderived DNA samples underwent a series of restriction enzyme digestion steps to first reduce genome complexity, and then to introduce adapter-compatible overhangs for SMRTbell library preparation. Enrichment of the desired loci was achieved by Cas9-mediated digestions of the SMRTbell libraries using CTG18.1 (Fig. 1b) and previously optimized FMR1-specific ${ }^{13}$ gRNAs. Digested templates were enriched by ligation to new hairpin adapters and purified using MagBeads. For control purposes we used a multiplexing approach by cotargeting, enriching, and isolating a fragile X syndrome-associated CGG triplet repeat located within FMR1, at the same time as the FECD-associated CTG18.1 locus.

\section{Analysis of SMRT sequencing reads shows expected target capture}

SMRTbell libraries were sequenced using a PacBio RSII instrument, generating reads of up to $7.1 \mathrm{~kb}$ in length. ${ }^{15}$ Each CCS read generated comprised multiple passes over a single DNA molecule due to the circular nature of the SMRTbell templates being sequenced. ${ }^{17}$ CCS reads were then mapped onto the human genome (hg19) and genome-wide coverage plots were generated (Supplementary Figure S1). Each sample-specific plot displayed clear peaks representative of on-target reads mapped to the captured loci on chromosomes 18 (CTG18.1) and X (FMR1). The average number of ontarget CCS reads generated, per sample, was 207 for the FMR1 loci and 897 for the TCF4 loci (Table 1). The FMR1 repeat region was successfully captured and analysis of the locus, as anticipated, did not detect any disease-associated repeat expansions (defined as $>55$ copies of the CGG repeat ${ }^{18}$ ) within our FECD cohort (Table 1). Off-target reads were observed for all samples. The most notable and consistent offtarget coverage peak was observed on chromosome 21 (Chr21: 19,639,348-19,639,367). We were able to retrospectively attribute this signal to a region with similarity to the TCF4-specific gRNA targeted region on Chr18: 53,252,023$53,252,042$. In total, four mismatches were detected between the TCF4-specific gRNA sequence and the nontargeted region on chromosome 21 (Supplementary Figure S2). Additionally, lower levels of off-target reads were found to consistently map to the centromeric region of chromosome 10:42,383,760$42,393,200$ (Supplementary Figure S1). However, this signal was determined to be mapping artifact due to the high levels of mismatches between the reads and reference genome (Hg19). All off-target reads generated were filtered as part of our selection and filtering strategy (see below) and hence did not affect analysis of TCF4 or FMR1-specific reads. 


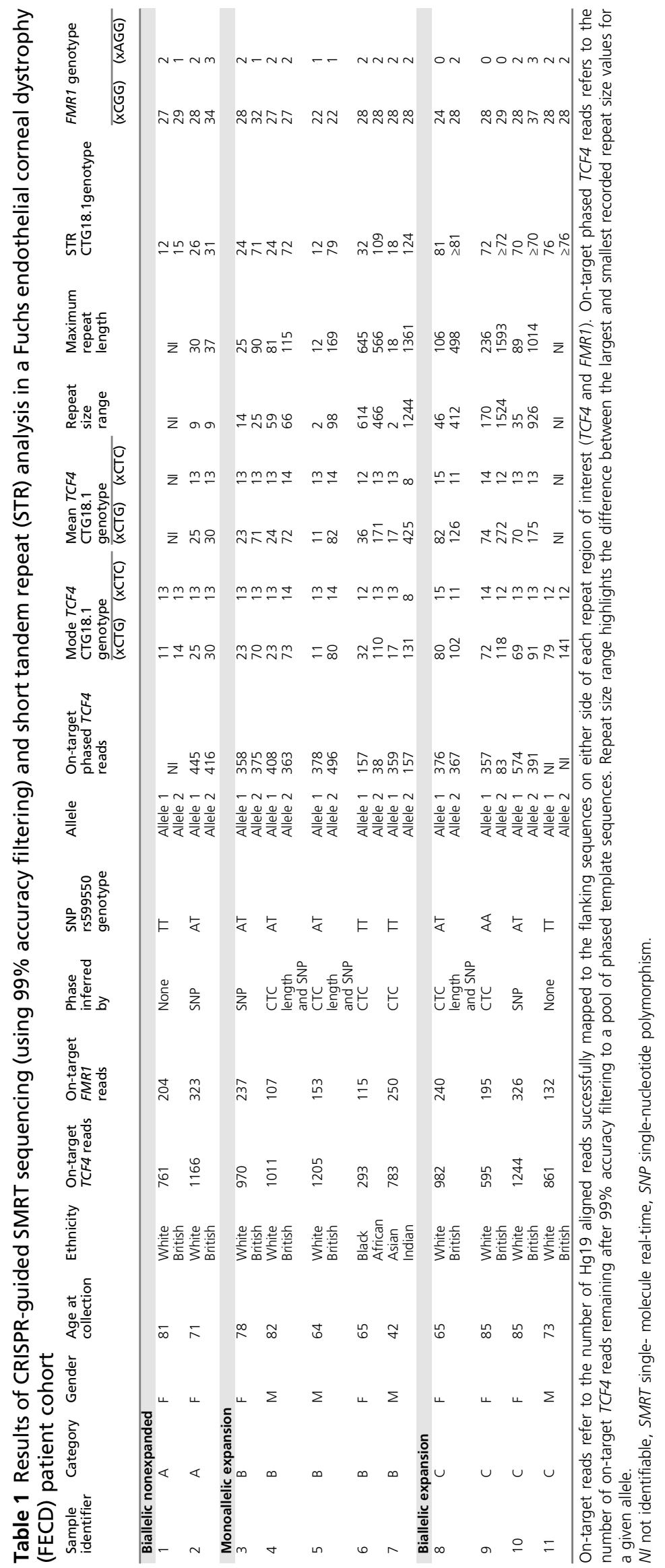


a
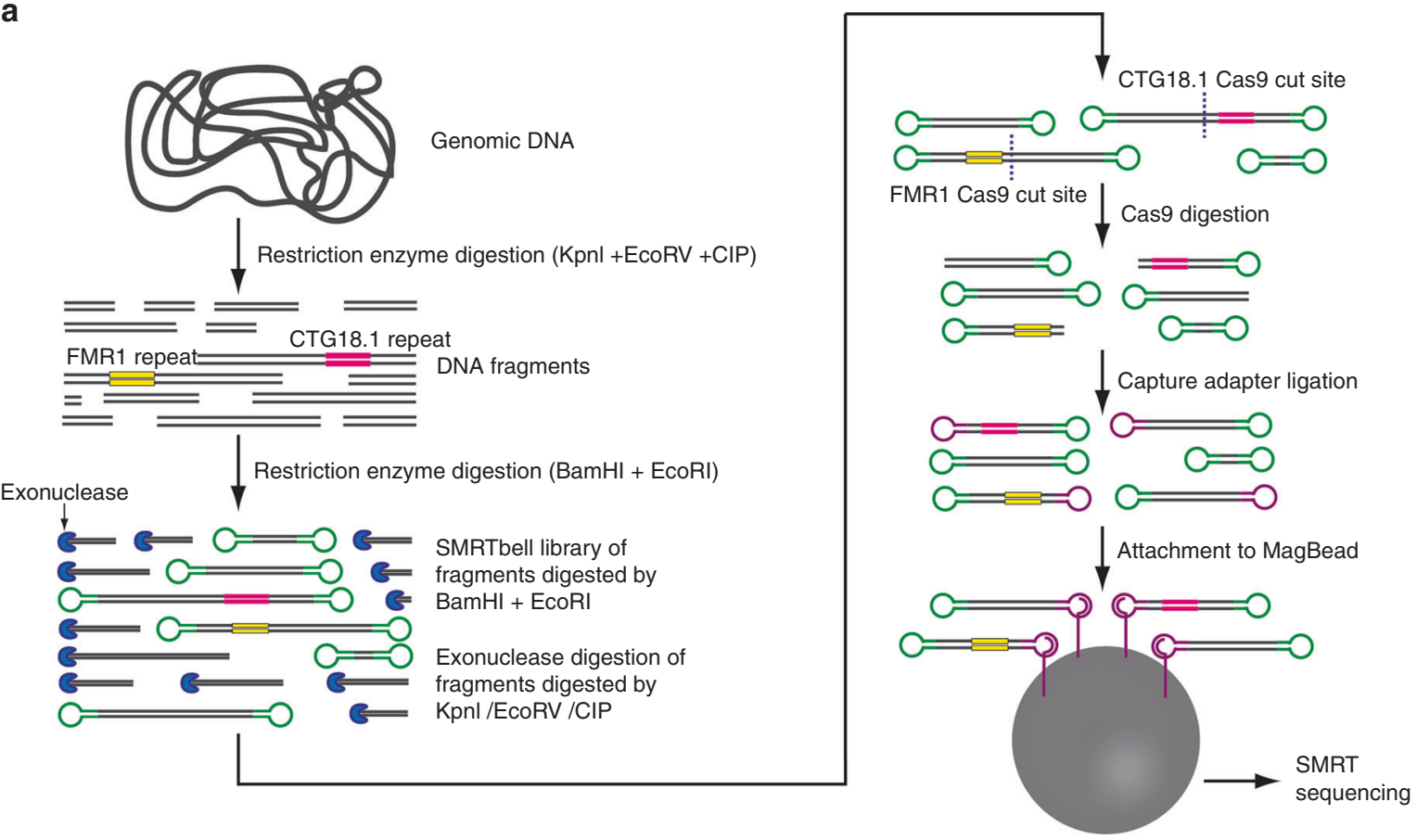

b

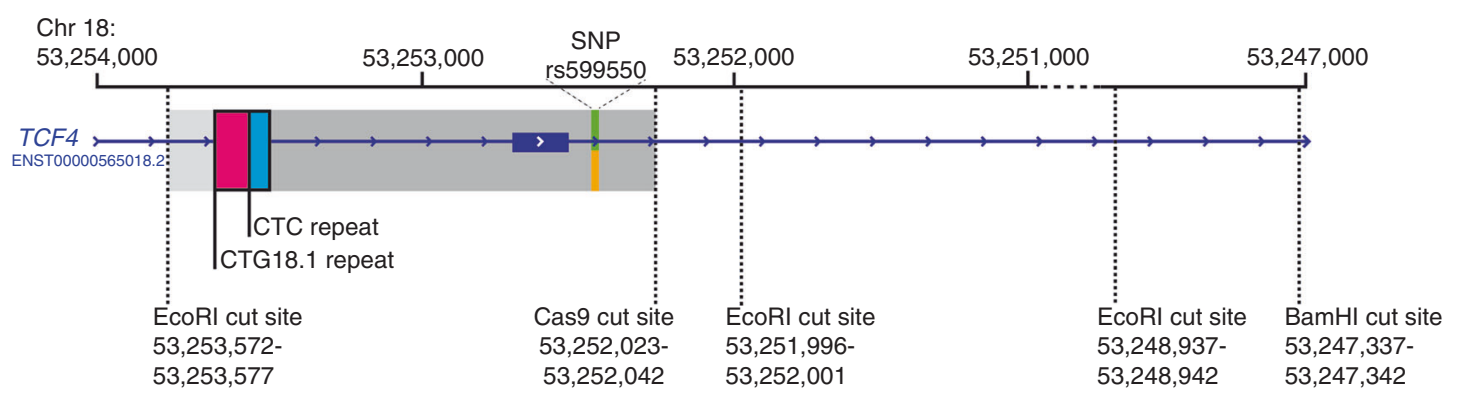

C

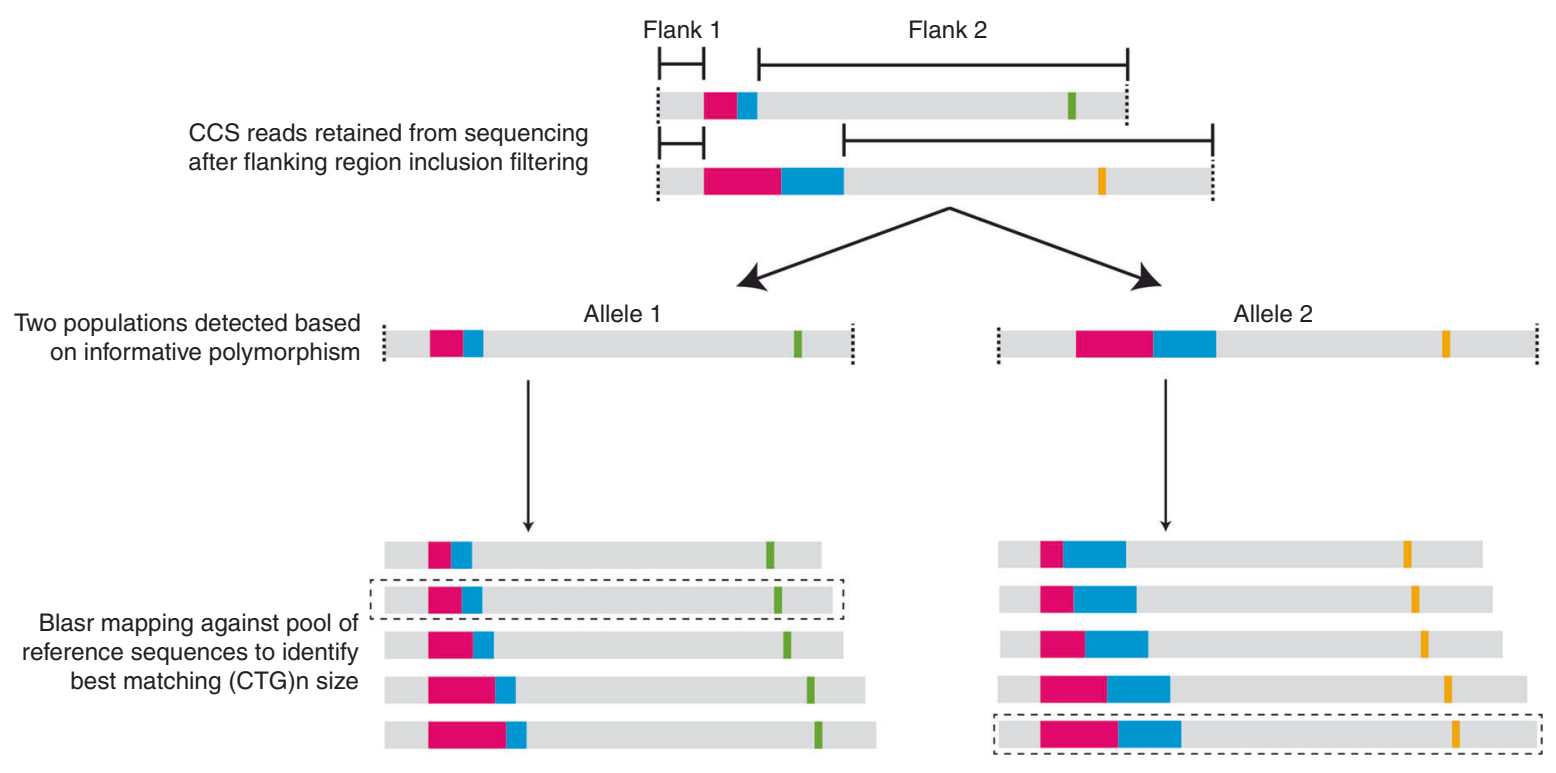


Fig. 1 Schematic of CRISPR-guided single-molecule real-time (SMRT) sequencing methodology, targeted capture design, and downstream analysis strategy for the CTG18.1 loci. (a) First, genomic DNA underwent a complexity reduction step by digestion with selected restriction enzymes not predicted to cut inside the target region(s); nontargeted fragments were subsequently degraded by exonuclease. Targeted loci, TCF4 CTG18.1 and FMR1 (positive control), are depicted as pink and yellow respectively. A SMRTbell (green) library was created after target loci were excised by EcoRI and BamHI. Guide RNAs (gRNAs) targeted specifically to sequence adjacent to the desired regions (TCF4 and FMR1) enabled Cas9 digestion. Cas9-digested SMRTbell fragments were ligated with engineered capture adapters (purple) and the fragments were attached to MagBeads. (b) EcoRI sites surrounding the CTG18.1 repeat element were identified for target capture. A gRNA Cas9 cut site was selected downstream of the CTG18.1 repeat (pink). Polymorphisms including a CTC repeat (blue) and single-nucleotide polymorphism (SNP) rs599550 (green/orange) were encompassed within the targeted region. (c) On-target read selection was performed by filtering reads that did not contain two flanking regions either side of the repeat locus ( $\geq 90 \%$ mapping required, not including repeat). Whenever possible CTC repeat length and/or SNP heterozygosity was used to phase circular consensus sequencing (CCS) reads. Once phased, CCS reads were mapped against a pool of reference sequences of all possible CTG18.1 repeat lengths. The reference sequence with the greatest similarity to each individual CCS read was used to infer the CTG repeat length.

\section{Selection and analysis of CTG18.1-derived SMRT sequencing reads}

This is the first time, to the best of our knowledge, that CRISPR-guided SMRT sequencing has been used to generate long-read sequencing data for the CTG18.1 locus and we therefore needed to develop an analysis pipeline to effectively select and interrogate CTG18.1-specific reads. Firstly, CCS reads were filtered to exclude those that did not encompass regions flanking the repeat (Fig. 1c). Next, a preliminary analysis step was performed to estimate the maximum repeat lengths, per sample, and to determine if informative polymorphisms were present within the flanking regions. Importantly, two commonly polymorphic markers were identified within flank 2 (Fig. 1b, c): a CTC repeat located immediately 3 ' to the CTG repeat of interest, and a polymorphic SNP (rs599550) $1320 \mathrm{bp}$ downstream of CTG18.1. The CTC repeat lengths in our cohort ranged from 8 to 15 copies and always included one CTT repeat interruption. When individuals were found to be heterozygous for either the length of this repeat or rs599550, we had the potential to phase reads. Within this cohort we were able to phase 9/11 samples on this basis (Table 1).

Subsequently, a more comprehensive mapping approach was employed to accurately determine repeat lengths. This involved mapping CCS reads to a customized pool of reference templates, devised after our initial TCF4 loci-specific analysis step, which comprised all possible combinations of CTG18.1 allele lengths, in addition to the previously determined polymorphic marker genotypes, when appropriate (rs599550 and CTC repeat; Fig. 1b and c). Only reads that achieved $\geq 99 \%$ similarity to a sequence within the customized pool of templates were included. Sized on-target reads were then visualized and histograms were generated to depict the range of CTG18.1 repeat lengths observed in each sample (Figs. 2 and 3; Supplementary Figure S3).

\section{CRISPR-guided SMRT sequencing of CTG18.1 improves genotyping accuracy and reveals that expanded CTG18.1 alleles behave dynamically}

Given that all samples included in this study had previously been genotyped for CTG18.1 by STR analysis we were able to make a direct comparison between the alternative methods.
Average allele lengths observed by CRISPR-guided SMRT sequencing for samples comprising nonexpanded CTG18.1 alleles (categories $\mathrm{A}[n=4$ alleles $]$ and $\mathrm{B}[n=5$ alleles $]$ ) were concordant with our previous STR analysis. However, a discrepancy of one or more repeat units per allele was noted between these differing methods. For example, sample 1 had previously been genotyped $12 / 15$ by STR analysis whereas CRISPR-guided SMRT sequencing results suggested a genotype of 11/14 (Table 1). These differences are likely in part attributed to variability in the size of the polymorphic CTC repeat located directly 3' of CTG18.1, given that the STR assay cannot distinguish differences in CTG18.1 length from variability in the length of flanking regions included within PCR amplimer. ${ }^{4}$ All sizing estimates calculated by STR analysis are based upon the reference genome (hg19), which contains 12 copies of the CTC motif. When this length deviates from 12 (e.g., sample 1), or indeed if any further insertions or deletions are present within the PCR amplified region, it will impede the accuracy of CTG18.1 sizing estimates provided by the STR assay. Data generated from category A samples (Supplementary Figure S3: Table 1) therefore exemplify that CRISPR-guided SMRT sequencing can effectively sequence biallelic nonexpanded CTG18.1 alleles and, importantly, the sequence level data generated provides improved levels of genotyping accuracy in comparison with STR analysis.

Importantly, much greater differences were observed between the STR and CRISPR-guided SMRT sequencing results for all expanded alleles analyzed (categories $\mathrm{B}[n=5$ alleles] and $\mathrm{C}[n=8$ alleles $]$ ). This is attributed to the CTG18.1 instability detected for all expanded CTG18.1 alleles sequenced using the CRISPR-guided SMRT sequencing method (samples 3-11; Table 1). The histograms presented in Figs. 2 and 3 and the dot plot presented in Fig. 4 illustrate this point. For category B samples, it is apparent that alleles comprising expanded copies of CTG18.1 ( $\geq 50$ copies) display greater levels of mosaicism than nonexpanded alleles $(<50$ copies) (samples 3-7; Fig. 2). Furthermore, levels of repeat length instability were found to increase with average allele length (Fig. 4; Table 1). For example, expanded alleles from samples 6 and 7 have mean repeat lengths of 171 and 425, repeat size ranges of 466 and 1244, and maximum repeat lengths of 566 and 1361, respectively. Whereas, samples 3, 4, 

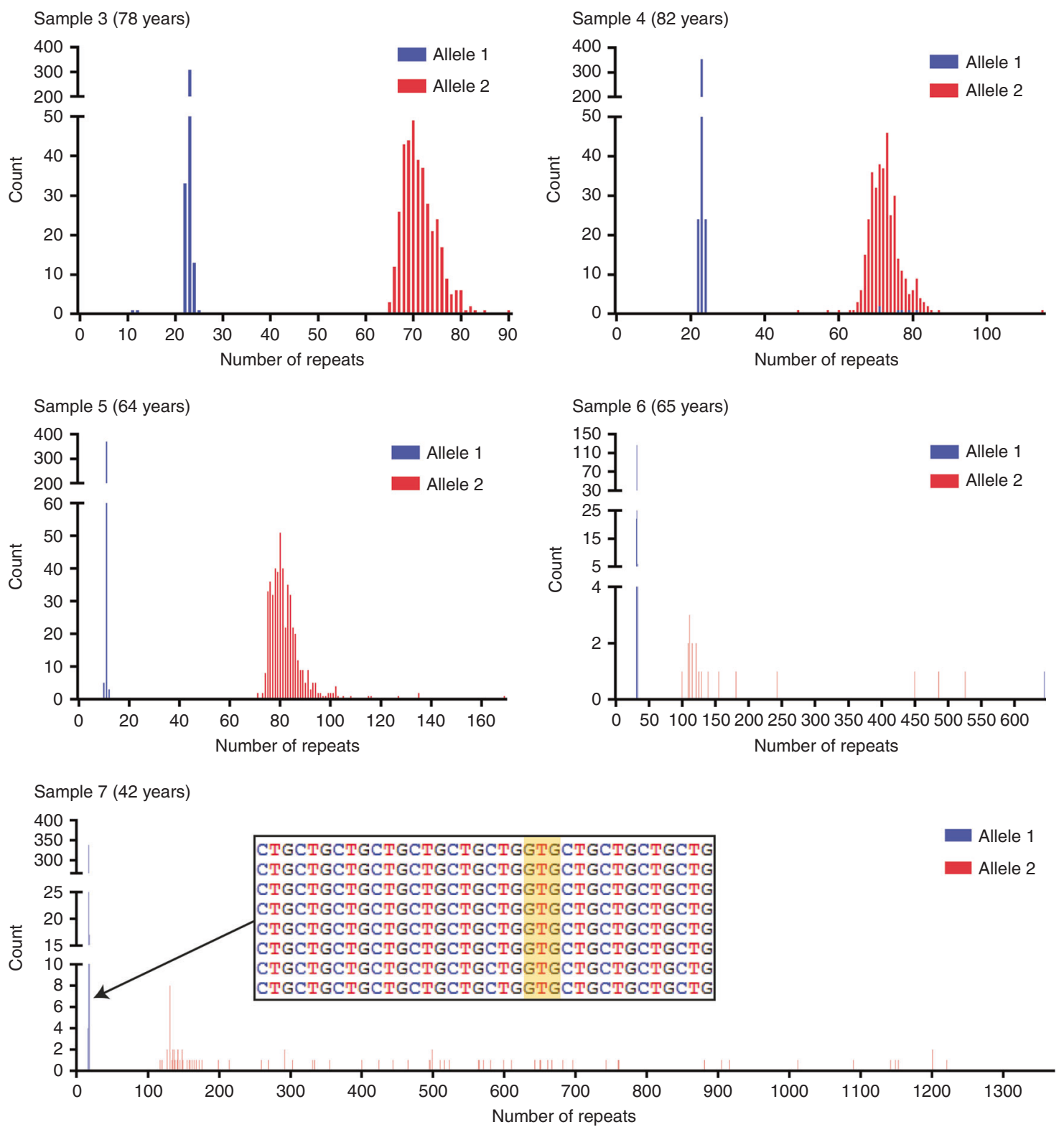

Fig. 2 Histograms to illustrate CTG18.1 repeat length distributions for samples harboring monoallelic expansions (category B). Histograms show CTG18.1 repeat length read counts after filtering circular consensus sequencing (CCS) reads with $\geq 99 \%$ similarity to the best matched reference sequence. All samples (3-7) could be phased using CTC repeat number and/or rs599550. A single base pair interruption was identified on a single nonexpanded allele (sample 7) by overlapping and visualizing aligned CCS reads (inset).

and 5 all have comparatively shorter average expanded repeat lengths $(71,72$, and 82$)$, display less diverse repeat size ranges $(25,66$, and 98$)$, and maximum repeat lengths detected, per sample, are lower (90, 115, and 169) (Table 1; Fig. 2; Fig. 4). Interestingly, the same pattern is also apparent for the phased samples 8, 9, and 10; (Fig. 3) where CTG18.1 repeat length instability positively correlates with the mean CTG18.1 length (Table 1; Fig. 4).

Importantly, CRISPR-guided SMRT sequencing also enabled us to comprehensively characterize biallelic expansions of CTG18.1 for the first time. Phased samples 8,9 , and
10 all harbored biallelic expansion with average repeat lengths of 82/126, 74/272, and 70/175 respectively. Previous STR analysis of the same samples could only provide sizing estimates for the smallest alleles present in each sample (Table 1). Additionally, analysis of phased CCS reads generated from sample 7 enabled us to identify a GTG repeat interruption within the nonexpanded allele comprising 17 repeats (Fig. 2, sample 7, inset). Both these examples highlight the power of the approach and advantages of using CRISPRguided SMRT sequencing over STR analysis to genotype CTG18.1. 

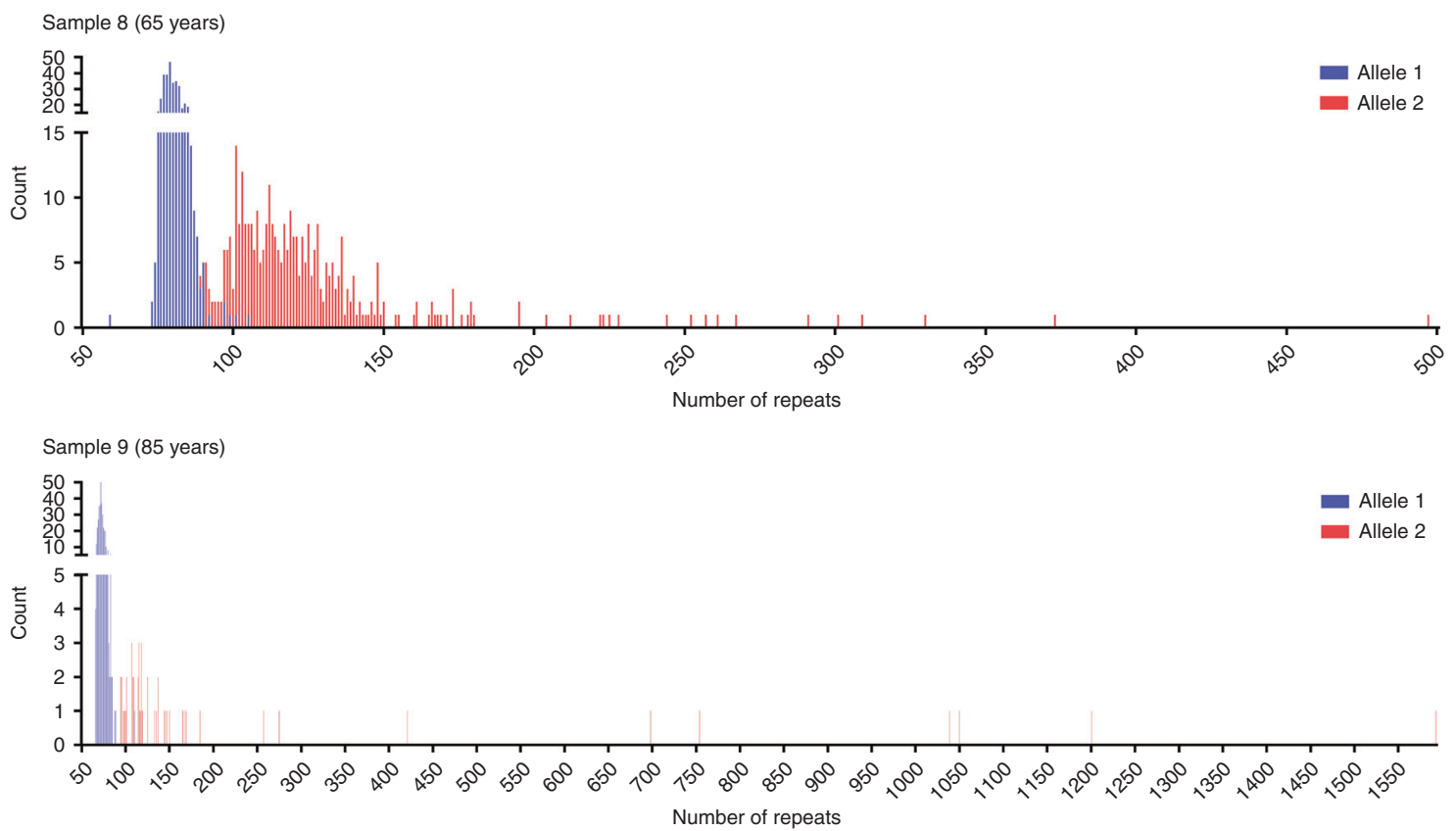

Sample 10 (85 years)
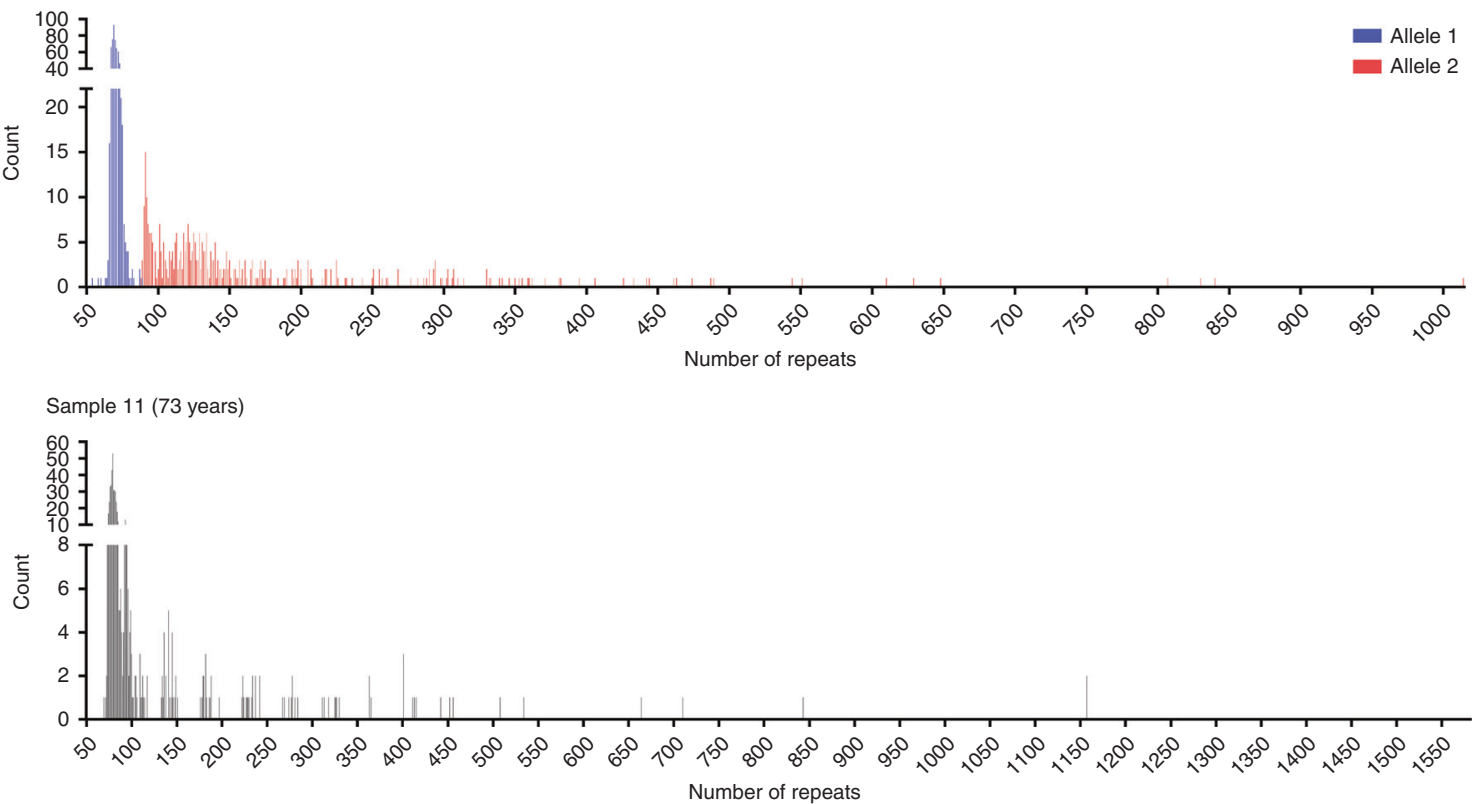

Fig. 3 Histograms to illustrate CTG18.1 repeat length distributions for samples harboring biallelic expansions (category C). Histograms show CTG repeat length read counts after filtering circular consensus sequencing (CCS) reads with $\geq 99 \%$ similarity to the best matched reference sequence. All sequenced alleles display repeat length instability. Samples 8-10 could be phased using CTC repeat number and/or rs599550. Sample 11 was unable to be phased; however, local maxima were indicative of two alleles being detected and sequenced.

\section{Stringency thresholds for read confidence requires compromise}

Low levels of potentially incorrectly phased CCS reads were observed using our CCS read selection and analysis strategy for samples 2, 4, 6, and 9 (Figs. 2 and 3 and Supplementary Figure S3). To determine if we could improve phasing confidence, we increased the percentage similarity filtering threshold used from $\geq 99 \%$ to $\geq 99.9 \%$ and reanalyzed all samples. As described previously, sized on- target reads were then visualized and histograms were generated to depict the range of CTG18.1 repeat lengths observed in each sample. This more stringent filtering approach resulted in only one single CCS read containing an expanded copy CTG18.1 remaining phased, potentially incorrectly, to an unexpanded allele. This read was generated from sample 6 and had been phased using the CTC motif only (Table 1). However, it was noted that using this approach inappropriately filtered all reads generated from sample 7 that 


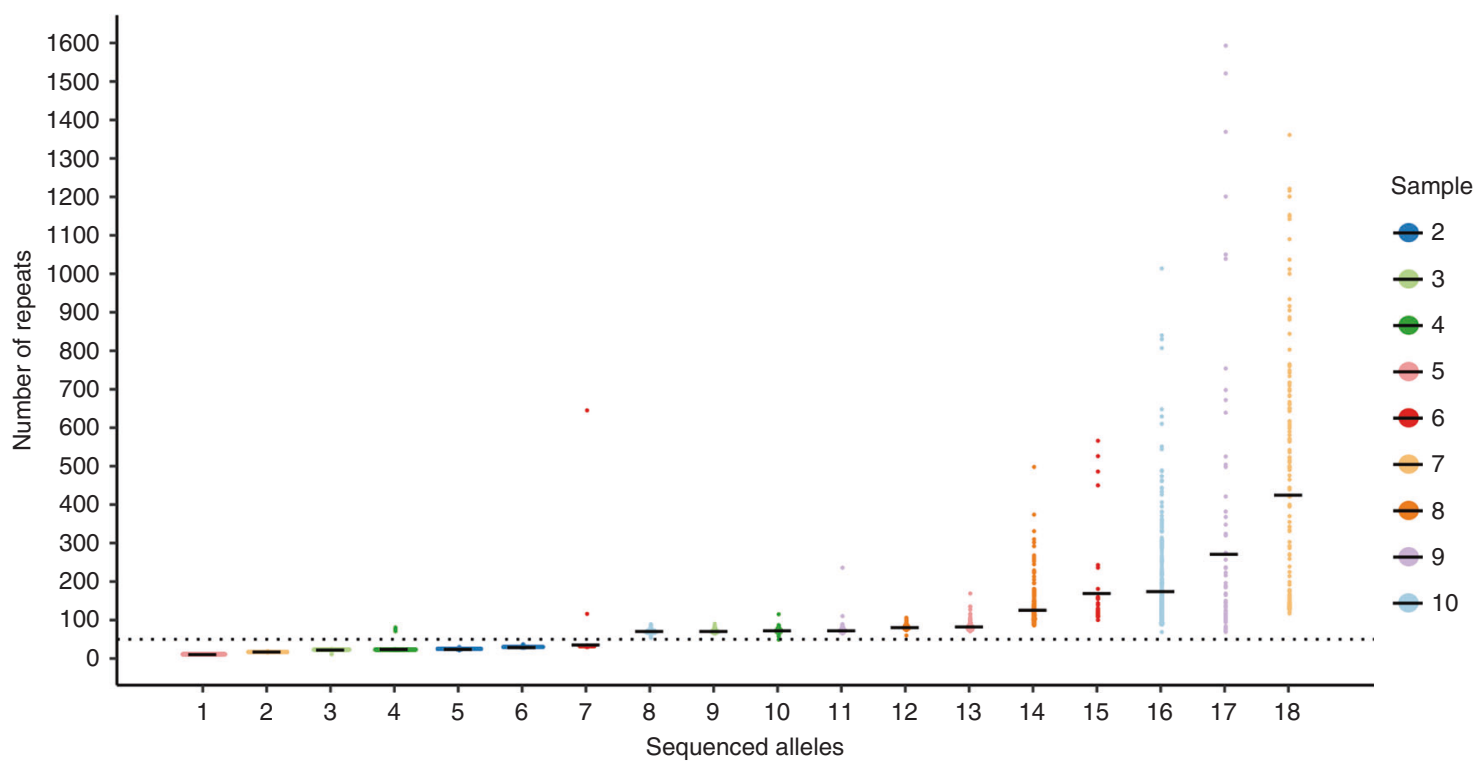

Fig. 4 CTG18.1 instability is correlated with repeat length. Dot plot highlights the change in magnitude of repeat instability observed across all phased alleles $(n=18)$. Samples are arranged in order of increasing mean allele length (plotted black lines represent mean per allele). Alleles are colored in accordance to sample numbers (2-10). A dashed line represents the disease-associated threshold of 50 repeats.

contain a repeat interruption, due to its absence from the reference template sequence to which the data is aligned (Fig. 2), highlighting the limitations of using such a stringent filtering threshold.

\section{DISCUSSION}

Here we demonstrate the utility of CRISPR-guided SMRT sequencing to interrogate the FECD-associated repeat motif, CTG18.1. Our proof-of-concept study illustrates that this amplification-free targeted enrichment approach, used in combination with long-read SMRT sequencing, can generate accurate sequencing data for clinically relevant samples. Furthermore, striking levels of repeat length instability and mosaicism were observed in our studied patient cohort, highlighting that size estimates provided by conventional genotyping assays (e.g., STR and Southern blot) do not provide a robust representation of the dynamic nature of this repeat element in its expanded state. Large-scale application of CTG18.1 locus-specific CRIPSR-guided SMRT sequencing will have great diagnostic utility and will enhance our understanding of CTG18.1 genotype diversity within FECD patient and control populations in addition to furthering our understanding of phenotype-genotype correlations for this common age-related disease.

In this study, we achieved an average of 327 reads per sample and had sufficient coverage to confidently determine CTG18.1 repeat lengths for all samples analyzed. On-target read depth was however notably lower for alleles comprising larger repeats, likely attributed to the inherent size difference between molecules being sequenced. Furthermore, we were able to phase reads for 9 of 11 samples analyzed. Future adaptations of this technology could consider modifying the size of the targeted flanking regions to maximize the likelihood of encompassing informative polymorphic markers to enhance phasing capabilities. To improve coverage, future efforts should focus on (1) gRNA design to reduce off-target Cas9 activity (e.g., off -target effect observed on Chr21; Supplementary Figures S1 and S2), (2) refinement of the pull-down stage of the protocol to reduce levels of SMRTbell molecules not cleaved by Cas9 being pulled down and sequenced, and (3) updating to the PacBio Sequel System 6.0 to enable increased multiplexing capacity at reduced cost with lower DNA input requirements. Importantly, reducing the required DNA input concentration for this method would also enable future analysis of corneal endothelial cell-derived DNA, which has the potential to provide insights into the tissue-specific nature of FECD. Furthermore, increasing multiplexing capacity will aid the diagnostic utility of the method. Notably, Tsai and colleagues have demonstrated that the method is already amenable to multiplexing across multiple genomic loci (bioRxiv: https://doi.org/10.1101/ 203919).

Instability of CTG18.1 repeats appears to consistently occur for the expanded alleles investigated in this study $(n=13)$. Furthermore, greater levels of instability are found with increased CTG18.1 length (Fig. 4) and were not found to correlate with donor age (Table 1). This phenomenon has previously been observed for other repeat expansion-mediated diseases. ${ }^{13,14,19,20}$ As anticipated, unexpanded alleles appeared to be relatively stable $(n=9)$. Prior to this study, all methods used to determine CTG18.1 repeat length, including STR analysis and Southern blotting, only provided crude sizing estimates and mode allele lengths. ${ }^{8,21}$ Our study highlights that although mode repeat lengths represent relatively reliable 
sizing estimates for up to approximately $\leq 80$ copies of the repeat element, they do not provide an accurate reflection of the true distribution of allele lengths when larger unstable expansions are present. This explains the discordant mode and mean allele lengths observed for alleles comprising $\geq 91$ copies of the repeat (Table 1). It also highlights that we should move away from considering CTG18.1 genotypes as stable entities in the expanded state and acknowledge that they are dynamic units. Future application of this technology has the potential to characterize the extent of tissue, and agedependent mosaicism, that will likely provide both diagnostic and biological insights into CTG18.1-mediated disease mechanisms.

In this study we detected a single GTG interruption within the CTG18.1 motif on a nonexpanded allele (sample 7). This interruption was readily detectable via visualization of aligned CCS reads. Intriguingly, up to $4.2 \%$ of unaffected aged populations harbor CTG18.1 expansions but it is not yet understood why these individuals remain unaffected. ${ }^{4,8}$ Interruptions of disease-associated repeat expansions have been shown to modulate phenotypic expressivity by interfering with DNA and RNA stability and/or downstream gain-offunction mechanisms. ${ }^{22-25}$ Future application of this sequencing technology could be used to address the lack of disease observed in unaffected individuals harboring presumed CTG18.1 expansions and/or atypical phenotype-genotype correlations observed within the patient population. However, the detection of interruptions within large expanded copies of the repeat motif will likely pose a challenge given the expected prominent levels of repeat length instability predicted to occur on such alleles.

There is great clinical need to develop new FECD treatment strategies. 5 Corneal transplantation is currently the only effective treatment option, and this relies upon an adequate supply of high quality donor material of which there is a global shortage. ${ }^{5,26}$ Given that expansion of CTG18.1 is associated with $>75 \%$ of FECD cases ${ }^{4,8-10}$ there is now much interest in developing gene-directed, CTG18.1-mediated treatment strategies. ${ }^{4,6,27}$ CRISPR-guided SMRT sequencing has the potential to aid the design and implementation of CTG18.1-targeted therapies in a clinical setting, providing a diagnostic framework for accurate and high-throughput CTG18.1 genotyping and informing genotype-dependent efficacy and outcomes. Given that the disease usually presents during the fifth to sixth decade of life, CRISPR-guided SMRT sequencing also has the potential to facilitate presymptomatic detection and identify patients suitable for future preventive therapies.

In conclusion, this custom application of CRISPR-guided SMRT sequencing has provided novel insights into levels of CTG18.1 length instability within an affected FECD cohort. Furthermore, this study provides a framework for improving molecular diagnostic accuracy for FECD, which is anticipated to become increasingly important as gene-directed therapies are developed for this common age-related disease. $^{4,27}$

\section{ELECTRONIC SUPPLEMENTARY MATERIAL}

The online version of this article (https://doi.org/10.1038/s41436019-0453-x) contains supplementary material, which is available to authorized users.

\section{ACKNOWLEDGEMENTS}

We thank all the patients for participating in this research. This work was funded by Fight for Sight Early Career Investigator Award (A.E.D.), Academy of Medical Sciences Springboard Award (A.E.D.), The National Institute for Health Research Biomedical Research Centre at Moorfields Eye Hospital National Health Service Foundation Trust and UCL Institute of Ophthalmology, Rosetrees Trust, Moorfields Eye Charity, and the National Eye Research Centre. P.L. was supported by GACR 17-12355 S and UNCE 204064. We thank Jenny Ekholm for reviewing the manuscript.

\section{DISCLOSURE}

Y-.C.T. is full-time employee and stock-holder of Pacific Biosciences (PacBio). T.A.C. was previously a full-time employee and is a stock-holder of Pacific Biosciences (PacBio). The other authors declare no conflicts of interest.

Publisher's note: Springer Nature remains neutral with regard to jurisdictional claims in published maps and institutional affiliations.

\section{REFERENCES}

1. Paulson H. Repeat expansion diseases. Handb Clin Neurol. 2018;147:105-123.

2. Chakraborty S, Vatta M, Bachinski LL, et al. Molecular diagnosis of myotonic dystrophy. Curr Protoc Hum Genet. 2016;91:9 29 21-29 29 19.

3. Lorenzetti DW, Uotila MH, Parikh N, Kaufman HE. Central cornea guttata. Incidence in the general population. Am J Ophthalmol. 1967;64:1155-1158.

4. Zarouchlioti C, Sanchez-Pintado B, Hafford Tear NJ, et al. Antisense therapy for a common corneal dystrophy ameliorates TCF4 repeat expansion-mediated toxicity. Am J Hum Genet. 2018;102:528-539.

5. Gain P, Jullienne R, He Z, et al. Global survey of corneal transplantation and eye banking. JAMA Ophthalmol. 2016;134:167-173.

6. Vedana G, Villarreal G Jr., Jun AS. Fuchs endothelial corneal dystrophy: current perspectives. Clin Ophthalmol. 2016;10:321-330.

7. Goldberg RA, Raza S, Walford E, et al. Fuchs endothelial corneal dystrophy: clinical characteristics of surgical and nonsurgical patients. Clin Ophthalmol. 2014;8:1761-1766.

8. Wieben ED, Aleff RA, Tosakulwong N, et al. A common trinucleotide repeat expansion within the transcription factor 4 (TCF4, E2-2) gene predicts Fuchs corneal dystrophy. PLoS ONE. 2012;7:e49083.

9. Nakano M, Okumura N, Nakagawa $H$, et al. Trinucleotide repeat expansion in the TCF4 gene in Fuchs' endothelial corneal dystrophy in Japanese. Invest Ophthalmol Vis Sci. 2015;56:4865-4869.

10. Xing C, Gong X, Hussain I, et al. Transethnic replication of association of CTG18.1 repeat expansion of TCF4 gene with Fuchs' corneal dystrophy in Chinese implies common causal variant. Invest Ophthalmol Vis Sci. 2014;55:7073-7078.

11. Soliman AZ, Xing C, Radwan SH, et al. Correlation of severity of Fuchs endothelial corneal dystrophy with triplet repeat expansion in TCF4. JAMA Ophthalmol. 2015;133:1386-1391.

12. Ebbert MTW, Farrugia SL, Sens JP, et al. Long-read sequencing across the C9orf72 'GGGGCC' repeat expansion: implications for clinical use and genetic discovery efforts in human disease. Mol Neurodegener. 2018;13:46 
13. Hoijer I, Tsai YC, Clark TA, et al. Detailed analysis of HTT repeat elements in human blood using targeted amplification-free long-read sequencing. Hum Mutat. 2018:39:1262-1272.

14. Schule B, McFarland KN, Lee $K$, et al. Parkinson's disease associated with pure ATXN10 repeat expansion. NPJ Parkinsons Dis. 2017;3:27.

15. Eid J, Fehr A, Gray J, et al. Real-time DNA sequencing from single polymerase molecules. Science. 2009;323:133-138.

16. Korlach J, Bjornson KP, Chaudhuri BP, et al. Real-time DNA sequencing from single polymerase molecules. Methods Enzymol. 2010;472:431-455.

17. Travers KJ, Chin CS, Rank DR, et al. A flexible and efficient template format for circular consensus sequencing and SNP detection. Nucleic Acids Res. 2010;38:e159.

18. Willemsen R, Levenga J, Oostra BA. CGG repeat in the FMR1 gene: size matters. Clin Genet. 2011;80:214-225.

19. Liu G, Leffak M. Instability of (CTG) $n *(C A G) n$ trinucleotide repeats and DNA synthesis. Cell Biosci. 2012;39:1262-1272.

20. Ardui $S$, Race $V$, Zablotskaya $A$, et al. Detecting AGG interruptions in male and female FMR1 premutation carriers by single-molecule sequencing. Hum Mutat. 2017;38:324-331.

21. Mootha VV, Gong X, Ku HC, Xing C. Association and familial segregation of CTG18.1 trinucleotide repeat expansion of TCF4 gene in Fuchs' endothelial corneal dystrophy. Invest Ophthalmol Vis Sci. 2014;55:33-42.

22. Braida C, Stefanatos RK, Adam B, et al. Variant CCG and GGC repeats within the CTG expansion dramatically modify mutational dynamics and likely contribute toward unusual symptoms in some myotonic dystrophy type 1 patients. Hum Mol Genet. 2010;19:1399-1412.

23. Landrian I, McFarland KN, Liu J, et al. Inheritance patterns of ATCCT repeat interruptions in spinocerebellar ataxia type 10 (SCA10) expansions. PLoS One. 2017:12:e0175958.
24. Santoro M, Masciullo M, Silvestri G, et al. Myotonic dystrophy type 1: role of CCG, CTC and CGG interruptions within DMPK alleles in the pathogenesis and molecular diagnosis. Clin Genet. 2017;92:355-364.

25. Kraus-Perrotta C, Lagalwar S. Expansion, mosaicism and interruption: mechanisms of the CAG repeat mutation in spinocerebellar ataxia type 1. Cerebellum Ataxias. 2016:3:20.

26. Golchet G, Carr J, Harris MG. Why don't we have enough cornea donors? A literature review and survey. Optometry. 2000;71:318-328.

27. Hu J, Rong Z, Gong X, et al. Oligonucleotides targeting TCF4 triplet repeat expansion inhibit RNA foci and mis-splicing in Fuchs' dystrophy. Hum Mol Genet. 2018;27:1015-1026.

Open Access This article is licensed under a Creative Commons Attribution 4.0 International License, which permits use, sharing, adaptation, distribution and reproduction in any medium or format, as long as you give appropriate credit to the original author(s) and the source, provide a link to the Creative Commons license, and indicate if changes were made. The images or other third party material in this article are included in the article's Creative Commons license, unless indicated otherwise in a credit line to the material. If material is not included in the article's Creative Commons license and your intended use is not permitted by statutory regulation or exceeds the permitted use, you will need to obtain permission directly from the copyright holder. To view a copy of this license, visit http://creativecommons.org/licenses/ by/4.0\%.

(c) The Author(s) 2019 